\title{
Video e-commerce: Large Scale Online Video Advertising based on user Preference
}

\author{
Gunavathie. M.A $\mathrm{A}^{\mathrm{a}}$, Kamalot Baavi.P ${ }^{\mathrm{b}}$, Saranya.J $\mathrm{J}^{\mathrm{b}}$, Pratheepa.P $\mathrm{P}^{\mathrm{b}}$, Roshini Suryadharshini.R ${ }^{\mathrm{b}}$ \\ ${ }^{a}$ Assistant Professor, Information Technology, Panimalar engineering college, Chennai, India \\ ${ }^{b}$ B.Tech, Information Technology, Panimalar engineering college, Chennai, India
}

Received: 28 March 2018; Accepted: 08 June 2018; Published: 08 September 2018

\begin{abstract}
An advertisement is a notice or announcement in a public medium promoting a product. Advertising plays significant role in the introduction of a new product in the market. It stimulates the people to purchase the product. In this paper, we propose a novel personalized Online Video Advertising System which is presented to recommend product ads from ecommerce sites to users of online video hosting. First we have to find a user preference, so we have to analyze each user's behavior of ecommerce sites. If the user wants to buy a product, user will spend more time to view the specification at the same time, user clicks that product more number of times to view the specification, price etc. These techniques are used to find the user preference. After collecting each user preference, we have to identify the semantic association between videos and products and construct the association between the key frames and products. A multi view deep learning approach is brought to view item features in different domains. When the User plays the video the user preferred advertisements are shown in the video in proper timestamps. Thus the advertisements are displayed only based on the user's preference and the user's will to purchase the desired product. This has been the key feature of our project. Maintaining privacy is one of the major problems for sharing personal information through social sites. Sharing video in social media may lead to unwanted problem and less privacy. As a result we need some tools for secured transmission. For satisfying this need, we propose a system called Adaptive Privacy Policy Prediction (A3P) to enhance privacy settings for user's data. This system provides a two-level framework for securing the data based on users browsing history on shopping site. It determines the best available privacy policy for users. Our solution relies on image classification which is associated with policies to upload images and also to user's social features. Hence by using this adaptive privacy policy our sharing of shopping and recommended videos can be secured to only desire people.
\end{abstract}

Index Terms: Frame split, video analysis, recommendation, user preferred advertisement, secured sharing.

(C) 2018 Published by MECS Publisher. Selection and/or peer review under responsibility of the Research Association of Modern Education and Computer Science.

\footnotetext{
* Corresponding author

E-mail address:

pratheepachiku@gmail.com, rahanish497@gmail.com 


\section{Introduction}

Video e-Commerce++, is presented for large scale online video advertising, which is able to exhibit appropriate product ads to particular users. In order to effectively mine the relationship among users, videos and products, an Incremental Co-Relation Regression model (ICRR), User Preference Diffusion (UPD), and Video Scene Importance model (VSIM) are proposed to ensure the product diversity, to alleviate the problems of data-sparsely and cold-start, and to portray the importance of video scene. Finally, Distributed Heterogeneous Relations Matrix Factorization (D-HRMF) is applied to conduct online advertising. It is worth to notice that ICRR is implemented to efficiently deal with new key frames or new products, which reduces the time complexity to meet the requirement of large scale online advertising.

In this proposed system, the video advertisements are only based on user preference. The User preference is found based on the user's behavior. Recently recommendations have become a hot topic and has been extensively studied [4][5] which suggest suitable products for users according to their preference. When user gets active on e-commerce sites, his activities are observed, till user logs out his account. User behavior analysis is done based on the frequently searched product and how long user watches the product. If the user wants to buy a product, the user will spend more time to view the specification at the same time user clicks that product more number of times to view the specification, price, review etc. These techniques are used to find the user preference. After finding the user preference, we embedded the user preferred product's advertisement in the video in proper time stamp. The relationship among each and every product is an essential factor for product recommendation [3]. We use collaborative filtering method to find out the user's rating or preference matrix [1,2].Using surf Detector algorithm, the video frames are classified based on products. Surf Detector is an algorithm, which extracts the some unique key points and descriptor from the product and frame and matches the key points to find the product in frame. Also users may face the problem of entering their personal details to register and share their content in the website. This is not recommended in many cases.

Sharing video may leads to less security of content [14].The user's privacy is not secured in this type of security [9], [12], [13], [15]. The existing privacy system is insufficient to solve the unique protection of video sharing [10], [11], [16] because the information carried within the video and their relevant social website are exposed. Hence we add upon the secured sharing technique to keep the contents secured and to maintain the privacy of the users.

\section{Existing System}

In the existing system, video based advertising system encountered several problems. Advertising systems recommend the same ads to all the users. It does not consider the user preference. Products and videos are increased dynamically, when the advertising systems face millions to billions of products and users, it cannot deal with large scale online advertising and conduct real time updating. Some of the most commonly used buttons online are the ones that allow users to skip the ad or simply close it altogether. If the advertisement system showing ads on the video while the user watching this is maybe an irrelevant ads for user and also user will get disturbed. Sometimes when people want to watch video, unwanted ads are placed over and over again on particular key frame. The ads are shown in improper time. Also it cannot handle the large amount of newly added videos, products and users.

\section{Proposed System}

The video advertisement only based on user preference. User preference find based on user behavior. When user will active his activities are observes, till user log out his account. User behavior analysis based on frequently search the product and how long user watches the product. User wants to buy a product, he spends more time to view the specification at the same he clicks the product more number of times to view the specification, price etc. These techniques used to find the user preference. After find a user preference, we 
embedded the user preference product's advertisement in video in proper timestamp. Using surf Detector algorithm, Classify video frames based on products. Surf Detector is a algorithm, which extracts the some unique key points and descriptor from the product and frame and matches the key points to find the product in frame.

\section{System Architecture}

The system architecture is shown in the figure 1 and the important characteristics in the system are explained below

\subsection{User Preference:}

The user logs in to the e-commerce site and searches the products, user's actions and behavior will be noted by admin and the time taken to view the specification, price, quality, the product added to cart and the liked products will be noted in the database. The products added to cart will be given highest priority. These techniques will help to find user's preference.

\subsection{Video Uploads:}

The videos are uploaded in the video site by the admin. The video is splitted into frames and the key frame is detected. Frame split is used to display the product in correct timestamp by considering the key-frame to display the product advertisement. It finds relationship between videos and products.

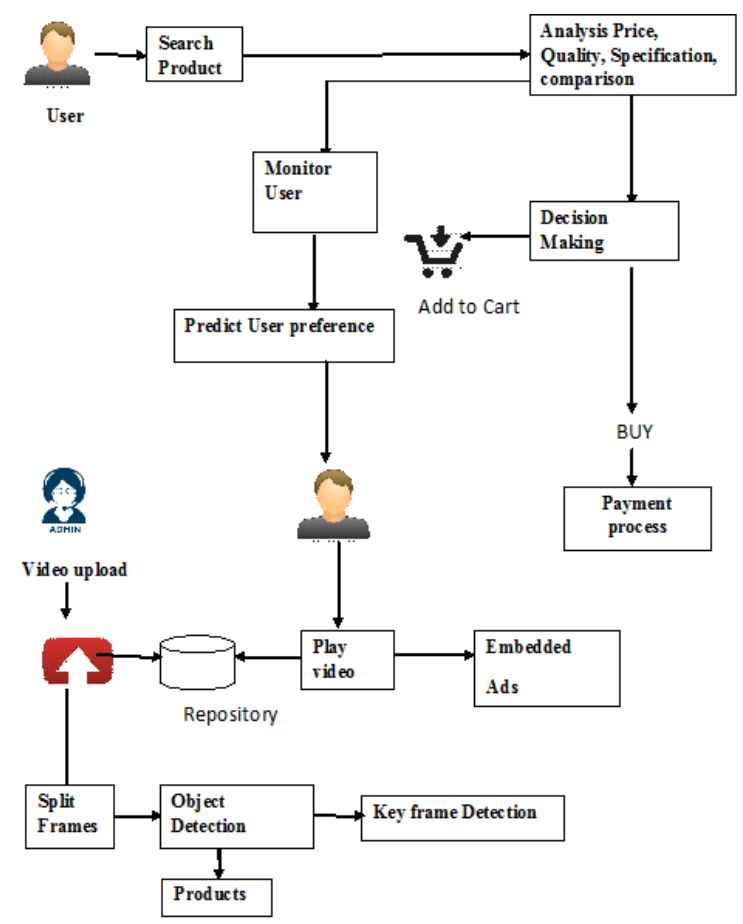

Fig.1. The System Architecture of video e-Commerce++ 


\subsection{User Interest:}

The user enters the details and creates an account. The user logs into his account and performs purchasing activity, his entire behavior will be noted until he logs out his account. Surf detection algorithm is used to group the unique points and find the match between product key points and frame key points. Highly preferred product will get displayed when the user watch the video. While seeing ad the user can click the buy button and buy the product by bank transaction.

\section{Modules}

The entire system is divided into five modules and modules descriptions are explained below

\subsection{Video uploads and Frame Splits}

This is the first module, here the video content providers or the admin login his account and uploads video based on category in Server. Video displays based on the frame rate. The frame rate is the number of frames or images that are projected or displayed per second. Frame rates are used in synchronizing audio and pictures. To show the ads, it finds a relationship between videos and products. By using ffmpeg tool, videos are splitted into frames.

\subsection{Product based key Frame Detection:}

Next we determine the semantic association between videos and products. Using surf Detector algorithm, the video is searched for the product by using the key points of the product. Surf Detector is an algorithm, which extracts the some unique key points and descriptor from the product and frame. It matches the product key points and frame key points. If the matches reach a particular threshold, the product is present in the image of the video. Surf Detector processes the all frames and finds the key frame for each product. The user registers his details and creates an account. The user watches the videos, which are uploaded by admin. Video sense system is introduced [8] to embed more contextually relevant ads in most appropriate positions in the video stream.

\subsection{User Preference Mining:}

In User Preference Mining, we determine the user preference based on the user's behavior. The user registers his details in e-commerce site and creates an account. User login his account and searches products based on his requirements. Once the user enters into his account, the user behaviors are observed till he logs out his account. User behavior is analyzed based on frequently search the product and how long the user watches the product. If the User wants to buy a product, he spends more time to view the specification at the same he clicks the product more number of times to view the specification, price etc. These techniques used to find the user preference. After finding the user preference the priority is provided for the products (Example: Priority will be given to newly updated product in the cart). When the user purchases a product it will go to payment process. User creates bank account and credit money. Product will be purchased based on his bank balance.

\subsection{Embedded ads on Video:}

In this module, the advertisements are embedded into the video in proper timestamps. After analyzing a user's preference, the particular user's preference is obtained from the e-commerce site. When the user selects any video, the system will verify whether is there any user preferred products available on the video. If it is present, it calculates the key frame and respective products ads will be displayed on the video in particular key 
frame. The relevance between users and videos are measured for high accuracy of advertising. For this private advertising system is provided [9] which recommend videos according to users willing.

\section{Surf Detection Algorithm}

The SURF method (Speeded Up Robust Features) is a fast and robust algorithm for local, similarity invariant representation and comparison of images.

The SURF detector algorithm focuses on blob-like structure in the image. This algorithm identifies the keypoints which are found at corners of objects. It detects blobs by convolving the source image. Gaussian's variance is used to normalize its response.

$$
\operatorname{DoH}(\mathrm{x}, \mathrm{y}, \sigma)=\frac{G_{x x}(x, y, \sigma) \cdot G_{y y}(x, y, \sigma)-G_{x y}(x, y, \sigma)^{2}}{\sigma^{2}}
$$

where

$$
G_{i j}(x, y, \sigma)=\frac{\partial N(0, \sigma)^{2}}{\partial i \cdot \partial j} * \operatorname{image}(x, y)
$$

Gaussian filters are used to get rid of noisy data by blurring the image. Gaussian blurring highlights image details at or near a single unique scale. The distinction in images are achieved by brighten the blobs found on a dark background versus darkening blobs found on bright background.

$$
\operatorname{Sgn}\left\{G_{x x}(x, y, \sigma)+G_{y y}(x, y, \sigma)\right\}=\left\{\begin{array}{l}
+1 \text { bright blob over dark background } \\
-1 \text { dark bob over bright background }
\end{array}\right.
$$

The SURF detector algorithm can thus be summarized by the following steps:

1. Form the scale - space response by convolving the source image using DoH filters.

2. Search for local maxima across neighbouring pixels and adjacent scales.

3. Interject the location of each local maxima obtained.

4. For each point of interest, return the DoH magnitude Laplacian's sign.

\section{Experiment and Analysis}

Video has provided a powerful new way to showcase products and online merchants are creating increasingly sophisticated videos to take advantage of viewer engagement. When you've invested time and money to create e-commerce videos, we want to realize a proper return on that investment. Still images have their place in e-commerce, but they are less effective than video when it comes to influencing a purchasing decision by online shoppers or telling your brand story. In image advertising system image sense is majorly used to study the methodology of automatically associating relevant ads [6][7] when the image appears in the video.

Video e-Commerce is used to handle large number of videos and products. Ads shown in improper time are avoided. Unwanted ads neglected and user preferred ads are displayed whenever the object is detected in the video. Same ads showed to all users. Hence Data sparsity problem is solved .Sharing of video contents may sometimes be insecure and may prompt to enter our personal details. The user privacy is not secure in this type of security [9],[12],[13],[15]. Thus our project uses (A3P) Adaptive Privacy Policy technique for secured sharing and to maintain our privacy. Hence the videos are shared with others, without entering any personal details of the user. The system has been implemented using FFmpeg, Java Server Page and MySQL. 


\section{Snapshots}

The implementation of the system has explained in the following snapshots.

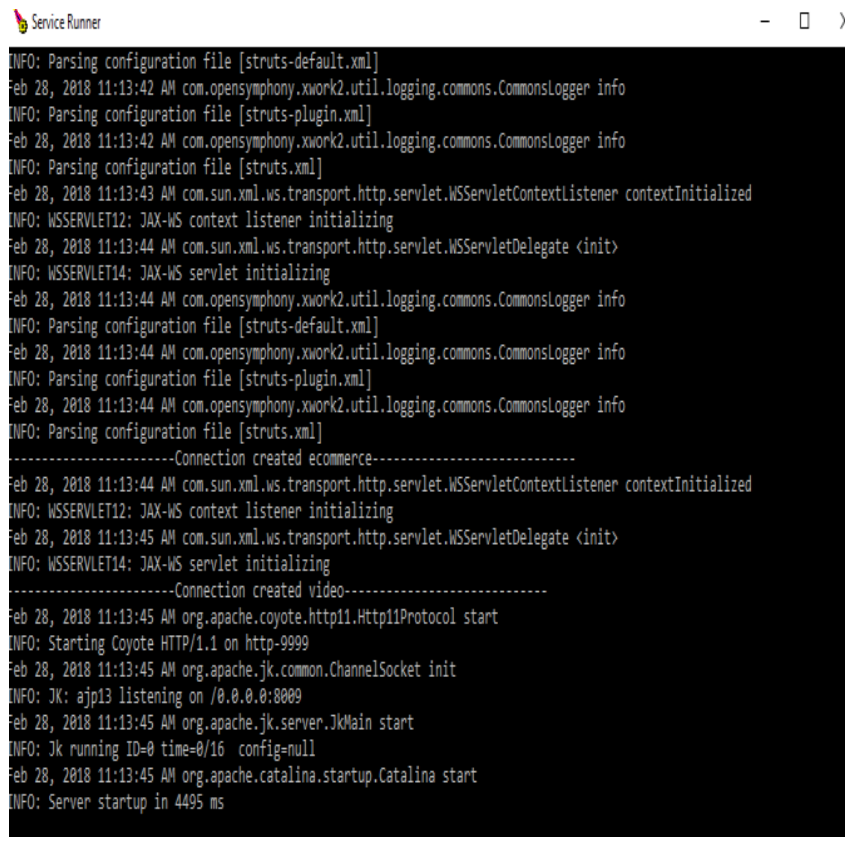

Fig.2. Start the Tomcat Server
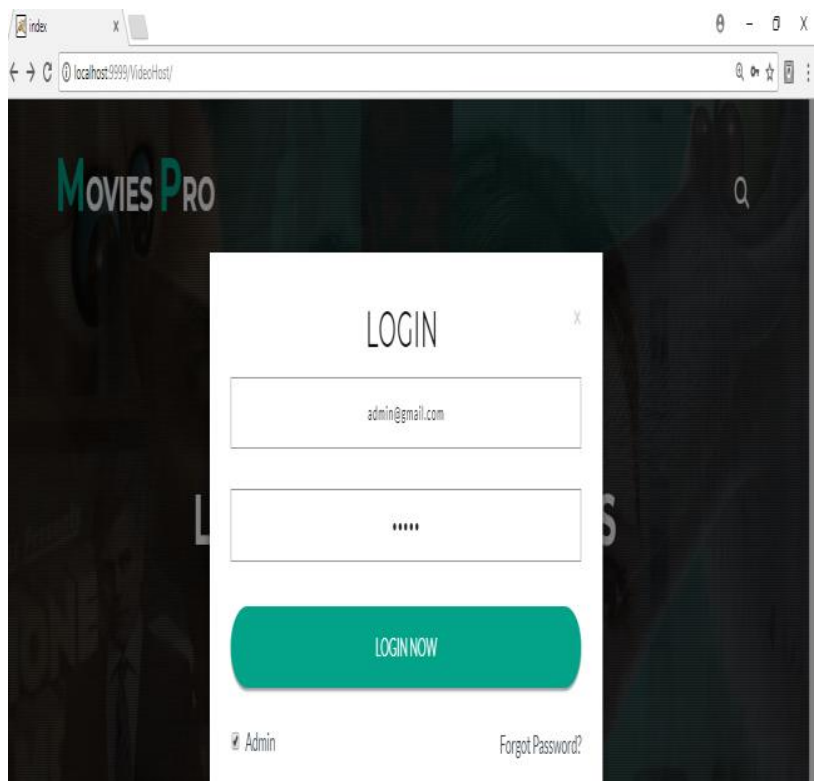

Fig.3. Once Server has Started to Run, the Admin Has to Login into the Video Website to Upload the Video. 


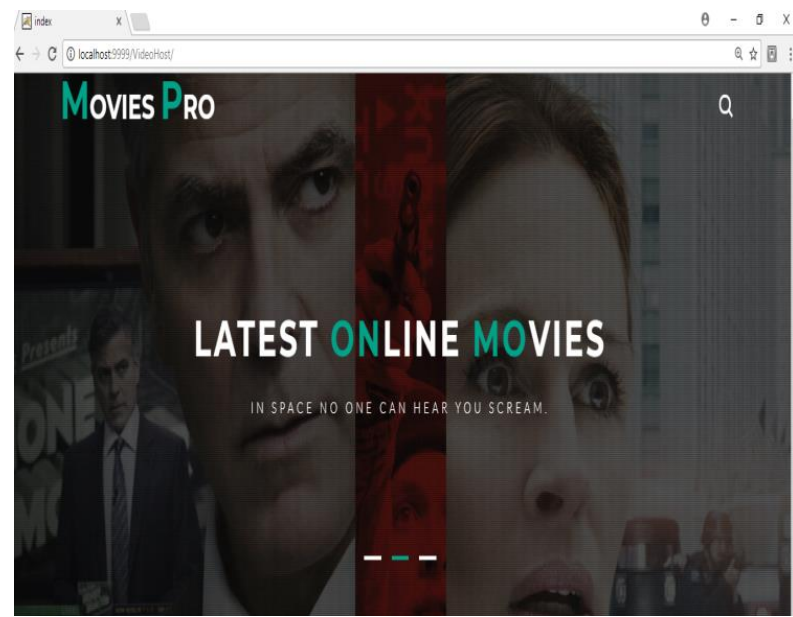

Fig.4. After the Login Procedure is Done, the Home Page Display Consists of Various Tabs (Like Feature Extraction, Frame, Upload, Sign-Out)

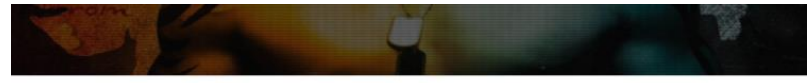

VIDEOUPLOAD

Choose Video

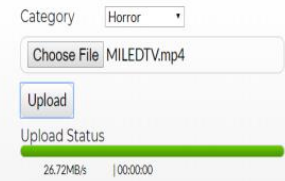

Fig.5. In the Video Upload Tab, the Category of the Video is Chosen and the Mp4 Video is Uploaded.

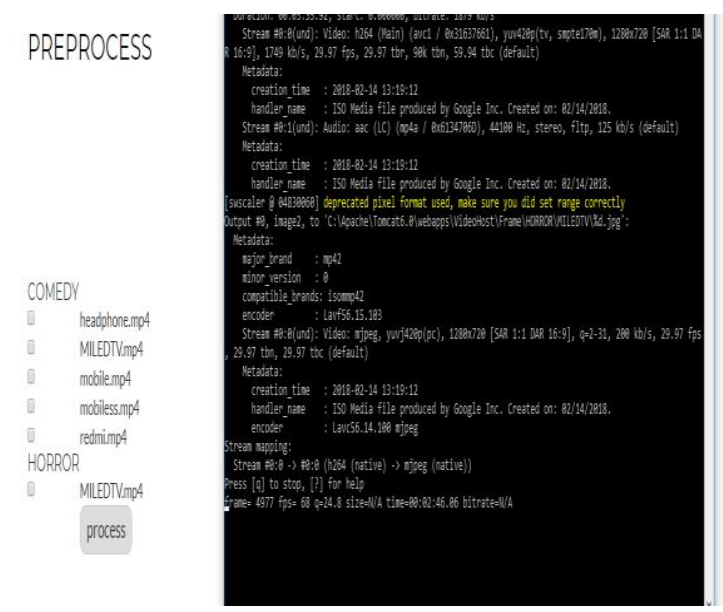

Fig.6. In Frame Tab, the Number of Frames Obtained from the Uploaded video is Displayed. 
KEYFRAME EXTRACTION

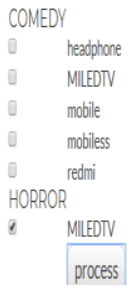

Fig.7. In feature Extraction, Each Key Frames Compared with the Appropriate Key Point and the Database Gets Updated.

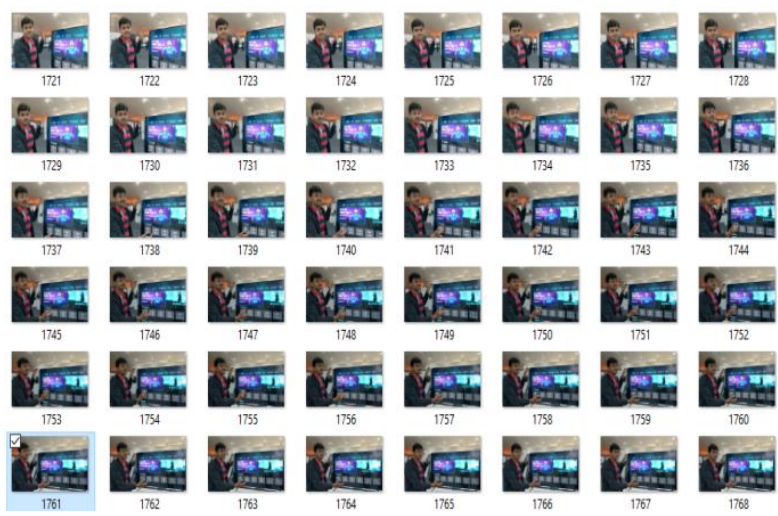

Fig.8. The Frames That Are Splitted and Saved in C Drive (the Ad Gets Displayed When the Object I.E. TV is Shown in the Frame.

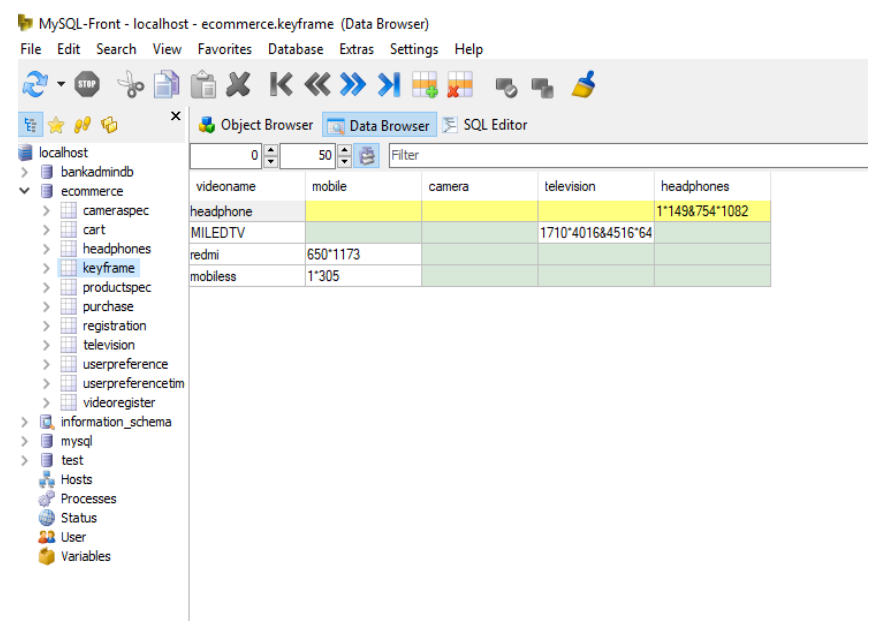

Fig.9. The MYSQL DB Displays the Total Number of Frames Up to Which the Object is Detected in the Video. 


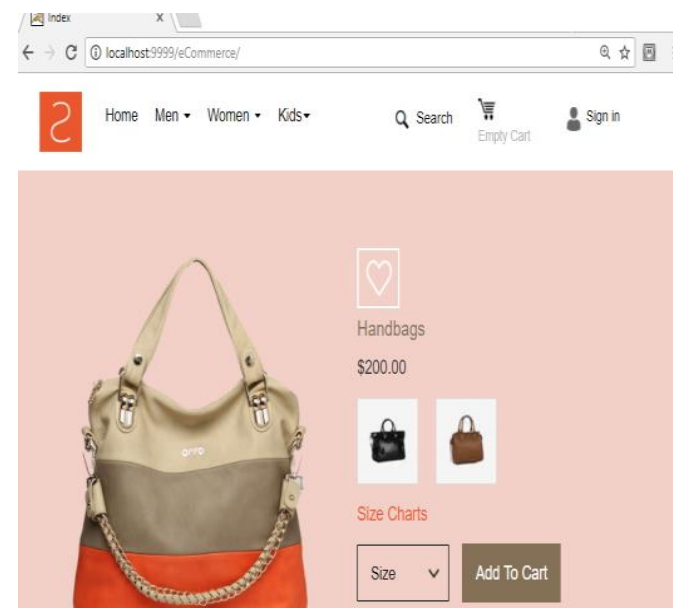

Fig.10. Shows the e-Commerce site, the User Can Login and Search the Necessary Items, All Activities of the User Is Noted by the Admin.

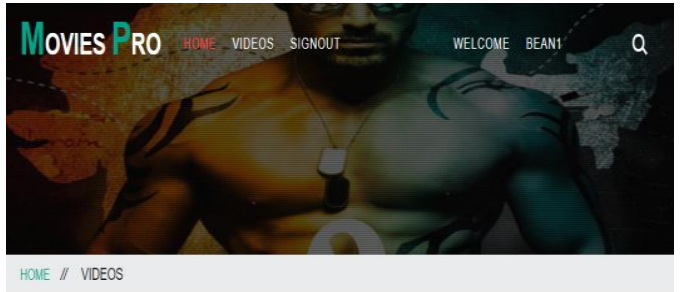

\section{COMEDY VIDEOS}

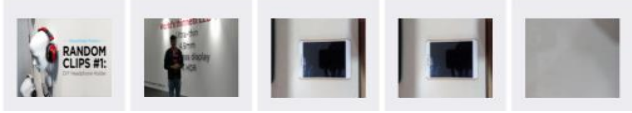

Fig.11. The user Logs in to the Video Site and Views the Video.

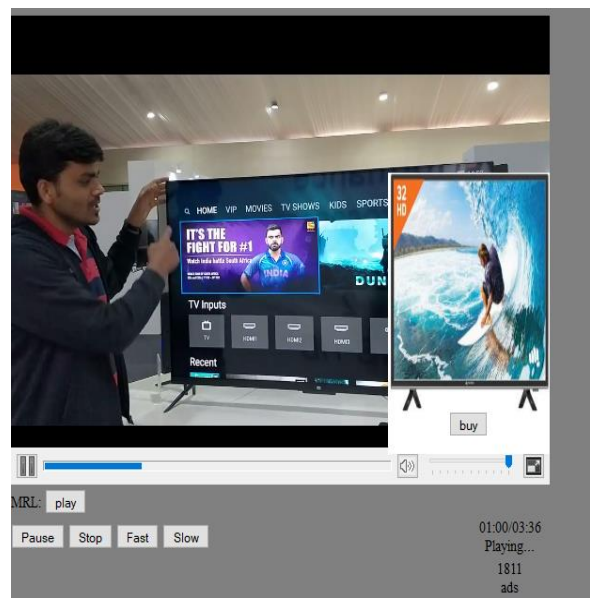

Fig.12. The Advertisement Gets Displayed When the User Preferred Object is Displayed in the Video. 


\section{Conclusion}

This paper video e-Commerce, performs large scale video advertising which provides appropriate product ads to users based on their preference. SURF detection algorithm is used to detect the points which are at corner of object. Providing privacy while sharing the video is an additional feature. We recommend Adaptive Privacy Policy Prediction(A3P) System which provides user free privacy setting.

\section{References}

[1] O. Sar Shalom, N. Koenigstein, U. Paquet, and H. P. Vanchinathan, "Beyond collaborative filtering: The list recommendation problem," in Proc. Int. World Wide Web Conf., 2016, pp. 63-72.

[2] A.M. Elkahky, Y. Song, and X. He, "A multi-view deep learning approach for cross domain user modeling in recommendation systems," in Proc. Int. World Wide Web Conf., 2015, pp. 278-288.

[3] T. Zhu, P. Harrington, J. Li, and L. Tang, "Bundle recommendation in ecommerce," in Proc. Int. ACM SIGIR Conf. Res. Devlop. Inf. Retrieval, 2014, pp. 657-666.

[4] J. J. McAuley, C. Targett, Q. Shi, and A. van den Hengel, "Image-based recommendations on styles and substitutes," in Proc. Int. ACM SIGIR Conf. Res. Develop Inf. Retrieval, 2015, pp. 43-52.

[5] A. Veit et al., "Learning visual clothing style with heterogeneous dyadic co-occurrences," in Proc. Int. Conf. Comput. Vis., 2015, pp. 4642-4650.

[6] T. Mei and X.-S. Hua, "Contextual internet multimedia advertising," Proc. IEEE, vol. 98, no. 8, pp. 1416-1433, Aug. 2010.

[7] T. Mei, X.-S. Hua, and S. Li, "Contextual in-image advertising," in Proc. ACM Int. Conf. Multimedia, 2008, pp. 439-448.

[8] T. Mei, L. Li, X.-S. Hua, and S. Li, "Image Sense: Towards contextual image advertising," ACM Trans. Multimedia Comput., Commun. Appl., vol. 8, no. 1, 2012, Art. no. 6.

[9] P. Zhou, Y. Zhou, D. Wu, and H. Jin, "Differentially private online learning for cloud-based video recommendation with multimedia big data in social networks," IEEE Trans. Multimedia, vol. 18, no. 6, pp. 1217-1229, Jun. 2016.

[10] S. Ahern, D. Eckles, N. S. Good, S. King, M. Naaman, and R. Nair. Over-exposed?: privacy patterns and considerations in online and mobile photo sharing. In Conference on Human factors in computing systems, pages 357-366. ACM, 2007.

[11] A. Besmer and H. Lipford. Tagged photos: concerns, perceptions, and protections. In CHI '09: 27th international conference extended abstracts on Human factors in computing systems, pages 4585-4590. ACM, 2009.

[12] L. Church, J. Anderson, J. Bonneau, and F. Stajano. Privacy stories: Confidence on privacy behaviors through end user programming. In Symposium on Usable Privacy and Security (SOUPS), 2009.

[13] H. Lipford, A. Besmer, and J. Watson. Understanding privacy settings in facebook with an audience view. In Proceedings of the Conference on Usability, Psychology, and Security, 2008.

[14] Y. Liu, K. P. Gummadi, B. Krishnamurthy, and A. Mislove. Analyzing facebook privacy settings: user expectations vs. reality. In 2011 ACM SIGCOMM conference on Internet measurement conference, IMC '11, pages 61-70. ACM, 2011.

[15] K. Strater and H. Lipford. Strategies and struggles with privacy in an online social networking community. In British Computer Society Conference on Human-Computer Interaction, 2008.

[16] C. A. Yeung, L. Kagal, N. Gibbins, and N. Shadbolt. Providing access control to online photo albums based on tags and linked data. In Social Semantic Web: Where Web 2.0 Meets Web 3.0 at the AAAI 2009 Symposium, 2009. 


\section{Authors' Profiles}

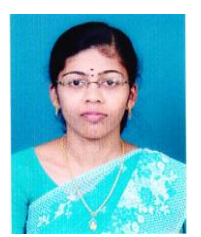

Gunavathie M. A was born in Madurai, Tamilnadu, India in 1988. She took her Bachelor's degree in Information Technology in Mepco Schlenk Engineering college. She completed her Masters degree in Information Technology from Madras Institute of Technology, Chennai. She is currently working as an Assistant professor in Panimalar Engineering College.

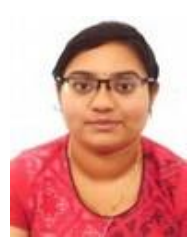

Kamalot Baavi.P was born in Chennai, India, in 1997. She received the B.Tech degree in Information Technology from Anna University, Chennai, Tamilnadu, India.

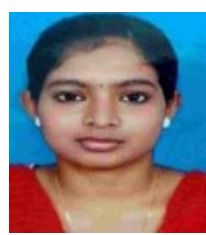

Saranya.J was born in Chennai, Indi in 1996. She received the B.Tech degree in Information Technology from Anna University, Chennai, Tamilnadu, India.

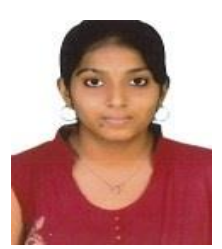

Pratheepa.P was born in Chennai, India, in 1997. She received the B.Tech degree in Information Technology from Anna University, Chennai, Tamilnadu, India.

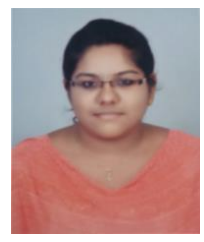

Roshini Suryadharshini. R was born in Chennai, India, in 1997. She received the B.Tech degree in Information Technology from Anna University, Chennai, Tamil Nadu, India.

How to cite this paper: Gunavathie. M.A, Kamalot Baavi.P, Saranya.J, Pratheepa.P, Roshini Suryadharshini.R,"Video e-commerce: Large Scale Online Video Advertising based on user Preference", International Journal of Education and Management Engineering(IJEME), Vol.8, No.5, pp.40-50, 2018.DOI: 10.5815/ijeme.2018.05.05 